\title{
The tree shrew is a promising model for the study of influenza B virus infection
}

\author{
Bing Yuan ${ }^{1,2 \dagger}$, Chunguang Yang ${ }^{3 \dagger}$, Xueshan Xia ${ }^{4 \dagger}$, Mark Zanin ${ }^{3}$, Sook-san Wong ${ }^{3}$, Fan Yang ${ }^{5}$, Jixiang Chang ${ }^{5}$, \\ Zhitong Mai ${ }^{3}$, Jin Zhao ${ }^{3}$, Yunhui Zhang ${ }^{1,2}$, Runfeng $\mathrm{Li}^{3}$, Nanshan Zhong ${ }^{3}$ and Zifeng Yang ${ }^{3,6^{*}}$
}

\begin{abstract}
Background: Influenza B virus is a main causative pathogen of annual influenza epidemics, however, research on influenza B virus in general lags behind that on influenza A viruses, one of the important reasons is studies on influenza B viruses in animal models are limited. Here we investigated the tree shrew as a potential model for influenza B virus studies.

Methods: Tree shrews and ferrets were inoculated with either a Yamagata or Victoria lineage influenza B virus. Symptoms including nasal discharge and weight loss were observed. Nasal wash and respiratory tissues were collected at 2, 4 and 6 days post inoculation (DPI). Viral titers were measured in nasal washes and tissues were used for pathological examination and extraction of mRNA for measurement of cytokine expression.

Results: Clinical signs and pathological changes were also evident in the respiratory tracts of tree shrews and ferrets. Although nasal symptoms including sneezing and rhinorrhea were evident in ferrets infected with influenza B virus, tree shrews showed no significant respiratory symptoms, only milder nasal secretions appeared. Weight loss was observed in tree shrews but not ferrets. V0215 and Y12 replicated in all three animal (ferrets, tree shrews and mice) models with peak titers evident on 2DPI. There were no significant differences in peak viral titers in ferrets and tree shrews inoculated with Y12 at 2 and 4DPI, but viral titers were detected at 6DPI in tree shrews. Tree shrews infected with influenza B virus showed similar seroconversion and respiratory tract pathology to ferrets. Elevated levels of cytokines were detected in the tissues isolated from the respiratory tract after infection with either V0215 or Y12 compared to the levels in the uninfected control in both animals. Overall, the tree shrew was sensitive to infection and disease by influenza B virus.
\end{abstract}

Conclusion: The tree shrew to be a promising model for influenza B virus research.

Keywords: Influenza B virus, Tree shrew, Ferret, Mouse, Animal model, List of Abbreviations.

\section{Highlights}

- Ferrets, tree shrews and mice were infected by influenza B virus strains of Yamagata or Victoria lineage isolated during our clinical surveillance. Viruses caused 100\% mortality in BALB/c mice.

- Viral replication and clinical signs of influenza were similar between tree shrews and ferrets, although

\footnotetext{
* Correspondence: jeffyah@163.com

${ }^{+}$Bing Yuan, Chunguang Yang and Xueshan Xia contributed equally to this work. ${ }^{3}$ State Key Laboratory of Respiratory Disease, National Clinical Research Center for Respiratory Disease, Guangzhou Institute of Respiratory Health, the First Affiliated Hospital of Guangzhou Medical University, Guangzhou, Guangdong 510120, People's Republic of China

${ }^{6}$ State Key Laboratory of Quality Research in Chinese Medicine, Macau University of Science and Technology, Avenida Wai Long, Taipa, Macau, People's Republic of China

Full list of author information is available at the end of the article
}

weight loss was only observed in tree shrews. These data show that tree shrews are a useful alternative to ferrets for studies of influenza B viruses, particularly considering their relatively small size, evolutionary relatedness to primates and lower cost compared to ferrets.

\section{Background}

Influenza B viruses (IBVs) are one of the main causes of seasonal influenza cases, accounting for up to $40 \%$ of total cases [1]. However, during the 2011/2012 and 2015/2016 influenza seasons in China, 53.7 and $52.2 \%$ of influenza notifications were due to IBV infections [2]. There are two lineages of IBVs currently in circulation; B/Victoria/2/1987-like (Victoria) and B/Yamagata/16/ 1988-like (Yamagata). Generally, IBVs cause a less severe 
respiratory disease compared to influenza A viruses (IAVs) [3]. However, IBVs cause more complications in children, including neurological, muscular [4] and cardiac-associated events [5]. Recent studies also showed that the antiviral drug Oseltamivir, the drug most commonly used to treat influenza, was less effective against IBV compared to IAV [6-8].

The tree shrew (Tupaia belangeri), is a small non-primate mammal that has been used as an animal model to study viral infection [9]. Tree shrews are a potentially promising model for the study of IBVs as they are more closely related to primates compared to ferrets, which are commonly used in influenza virus research $[10,11]$. Tree shrews are also smaller than ferrets, easier to handle and more cost-effective. Unlike mice, tree shrews display symptoms including fever, rhinitis and mild pneumonia post inoculation with IAVs, and show a distribution of $\alpha 2,3$-linked and $\alpha 2,6$-linked sialic acids in their respiratory tract similar to humans $[10,12]$. As such, here we conducted a study to determine if the tree shrew is a useful animal model for the study of IBV infection. We compared the tree shrew to two animal models commonly used in influenza research; ferrets and mice, using two strains of IBVs from Yamagata and Victoria lineages that were isolated from clinical specimens. Overall, we found the tree shrew to be sensitive to IBV inoculation, displaying a clear symptomology, viral shedding and seroconversion, thus demonstrating their utility as a model for future studies of IBVs.

\section{Materials and methods}

\section{Cells and viruses}

IBVs B/Guangzhou/0215/2012 (Victoria-like, V0215) and B/ Guangzhou/12/2016 (Yamagata-like, Y12) were obtained from the State Key Laboratory of Respiratory Disease of Guangzhou Medical University. These viruses were isolated during our previous study of IBVs isolated from human cases (partial data was published in Chinese) [13]. Briefly, our study of 8 viruses in the mouse model revealed that only the V0215 and Y12 viruses were capable of replication in the mouse model without prior adaptation. Therefore, these viruses were selected for this present study. Madin-Darby canine kidney (MDCK) cells (American Type Culture Collection) were cultured in Dulbecco's Modified Eagle's Medium (Gibco) supplemented with 10\% fetal bovine serum. All the viruses were cultured in MDCK cells cultures in DMEM without bovine serum and containing $1 \mu \mathrm{g} / \mathrm{mL}$ of L-(tosylamido-2-phenyl) ethyl chloromethyl ketone (TPCK) treated trypsin (Sigma). Virus titers were determined via the $50 \%$ tissue culture infective dose $\left(\mathrm{TCID}_{50}\right)$ method using MDCK cells and the Reed-Muench method [14].

\section{Animals}

Male tree shrews weighing 100 to $155 \mathrm{~g}$ and special-pathogen-free female six-week-old BALB/c mice were obtained from the Animal Experimental Centre of Kunming Medical University. Female ferrets weighing 600 to $700 \mathrm{~g}$ were obtained from Wuxi Coral Reef Biotechnology Co., Ltd. (Wuxi city, Jiangsu province, China). Ferrets and tree shrews were confirmed to be seronegative by virus neutralization assay to V0215 and Y12 viruses prior to commencement of experiments. All the animals were fed in IVC cages in a BSL-2 animal house at the Animal Experimental Centre of Kunming Medical University. Animals were acclimatized to their housing for 2 days prior to use in these studies.

Animals were divided into four groups; Group One comprised of ten tree shrews and ten ferrets. These animals were implanted with transponder microchips (IPTT-300, Bio Medic Data Systems Inc., Seaford, Delaware) to allow daily monitoring of body temperature for 5 days prior to intranasal inoculation with $1.0 \times 10^{6} \mathrm{TCID}_{50}$ of V0215 (five ferrets and five tree shrews) or Y12 (five ferrets and five tree shrews) in $250 \mu \mathrm{L}$ of phosphate buffered saline, $\mathrm{pH} 7.4$ (PBS). After inoculation, body weight and temperature were measured daily and nasal washes were collected using $1 \mathrm{~mL}$ of PBS at 2, 4 and 6 days post inoculation (DPI) to measure viral titers. Serum were collected 21 DPI to measure influenza-specific antibody titers. Seroconversion of the animals was tested by $\mathrm{HI}$ assays [15].

Group Two comprised of seven ferrets and 21 tree shrews. Three ferrets and nine tree shrews were inoculated with $1.0 \times 10^{6} \mathrm{TCID}_{50}$ of V0215 or Y12 and one ferret and three tree shrews were intranasally inoculated with equal PBS as negative controls. At 2, 4 and 6 DPI one ferret and three tree shrews inoculated with each virus were killed to collect respiratory tissues for pathology studies and real time PCR. The animals inoculated with PBS were killed at 6 DPI.

Group Three consisted of 15 mice. Five mice were intranasally inoculated with either $1.0 \times 10^{6} \mathrm{TCID}_{50}$ of V0215 or Y12 or equal PBS. These mice were observed for 15 days for mortality and morbidity.

Group four consisted of 35 mice. 15 mice were intranasally inoculated with $1.0 \times 10^{6} \mathrm{TCID}_{50}$ of either virus in $50 \mu \mathrm{L}$ of PBS and five were inoculated with equal PBS as a negative control. At 2, 4 and 6DPI five mice inoculated with either V0215 or Y12 virus were killed and lungs were collected for pathology studies and real time PCR. The five mice inoculated with PBS were killed at 6DPI and lungs were collected for pathology studies.

\section{Quantification of mRNA}

Tissues were homogenized and total RNA was extracted using Trizol reagent (Invitrogen) according to manufacturer's instructions. Reverse transcription was performed using PrimeScript RT Master Mix kit (TAKARA) according to manufacturer's instructions. Real-time PCR was conducted using TB Green premix Taq II (TAKARA) to measure cytokine 
and chemokine mRNA. Glyceraldehyde-3-phosphate dehydrogenase (GAPDH) was used as an internal reference gene. The primers were designed with Primer Primier 5.0 (Additional file 1: Table S1).

\section{Histology}

The tissues isolated from the respiratory tract were first fixed with $10 \%$ neutral buffered formalin, embedded in paraffin, sectioned at $3 \mu \mathrm{m}$ and stained with hematoxylin and eosin (H\&E) after dehydration, the pathological sections were observed and photographed under a microscope (Nikon, ECLIPSE Ni).

\section{Statistical analysis}

Data were analyzed using SPSS (version 13.0). The data were expressed as mean \pm standard deviation of the mean (SD). Significance was determined by single-tailed student's t-test when comparing two groups, Multiple group comparisons were performed via one-way-ANOVA test base on variance homogeneity. $p<0.05$ was considered significant.

\section{Results}

Influenza B viruses replicated in all animal models, causing lethal infections in mice

In our previous study we isolated IBVs from patients at the First Affiliated Hospital of Guangzhou Medical University [13]. As part of this study, we used 8 IBV strains to inoculate mice and we discovered two IBVs, V0215 and Y12, that caused severe disease without prior adaptation. In this current study we compared the susceptibility of tree shrews to ferrets and mice infected with V0215 and Y12 to determine the utility of tree shrews as models for future IBV studies. V0215 and Y12 replicated in all three animal models with peak viral titers evident on 2DPI (Fig. 1). There were no significant differences in titers between V0215 and Y12 inoculated ferrets, however, viral titers were significantly higher in tree shrews and mice inoculated with Y12 compared to V0215 at both 2 and 4DPI (Fig. 1a, b, c). There were no significant differences in peak viral titers in ferrets and tree shrews inoculated with Y12 at 2 and 4DPI, although viral titers were detected at 6DPI in tree shrews but not in ferrets. There was no mortality in tree shrews or ferrets and no significant differences in body temperature were observed in tree shrews or ferrets inoculated with either virus (Fig. 2a and b). Mean tree shrew body weight decreased post inoculation until 7DPI, with an overall mean weight loss of $10 \%$ (Fig. 2c). Weight loss was not detected in inoculated ferrets (Fig. 2d). Mice inoculated with either V0215 or Y12 began to lose weight at 2DPI and all mice were killed by 8DPI (Fig. 3). There were no significant differences in weight loss or mortality between mice inoculated with V0215 or Y12 (Fig. 3).

Seroconversion was observed in all tree shrews and ferrets. The antibody titer in ferrets and tree shrews inoculated with V0215 was significantly greater compared to Y12-inoculated animals $(p<0.05)$, indicating that the immunogenicity of V0215 was greater than Y12 (Table 1). Interestingly, HI titers measured in ferrets inoculated with either V0215 or Y12 were greater compared to tree shrews inoculated with those same viruses.

\section{Expression of cytokine and chemokine mRNA}

Inflammation was observed in tissues of the respiratory tract obtained from tree shrews, ferrets and mice inoculated with either V0215 or Y12. Increased amounts of IL-6, IL-10, IP-10, TNF- $\alpha$ and TGF- $\beta$ mRNA were detected at 2, 4 and 6DPI in tissues of the respiratory tract obtained from tree shrews (Fig. 4). V0215 elicited significantly greater expression of IL-6 and IP-10 mRNA at 2DPI compared to Y12 (Fig. 4a and d). In ferrets, amounts of IL-6, IL-8 and IP-10 mRNA were increased whilst IL-10 mRNA amounts were not appreciably increased post inoculation (Fig. 5). It should be noted that
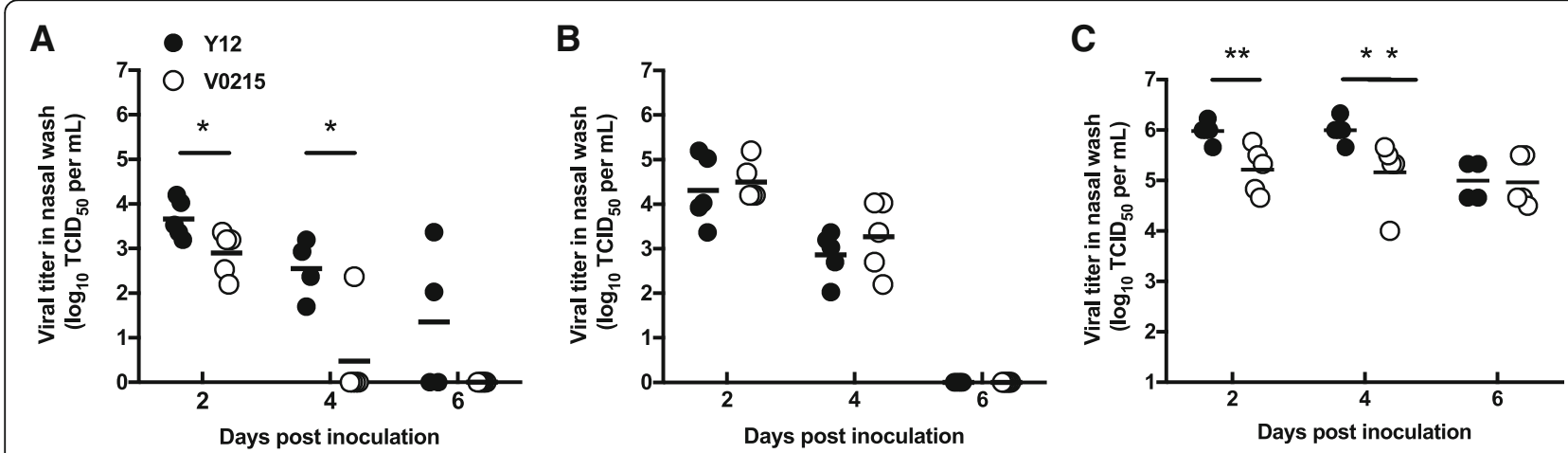

Fig. 1 Influenza B viruses can replicate in tree shrews, ferrets and mice. Tree shrews (a), ferrets $(\mathbf{b})$ and mice $(\mathbf{c})$ were inoculated with $1.0 \times 10^{6} \mathrm{TCID} \mathrm{D}_{50}$ of virus intranasally. At 2, 4 and 6 days post inoculation (DPI), nasal washes were collected and virus titers were determined by tissue culture infectious dose 50\% (TCID 50 ) assay in Madin Darby canine kidney (MDCK) cells $\bullet$ Yamagata strain B/Guangzhou/12/2016 (Y12), oVictoria strain B/Guangzhou/0215/ 2012 (V0215). Lines indicate means. ${ }^{* *} p<0.01,{ }^{*} p<0.05$ 
A

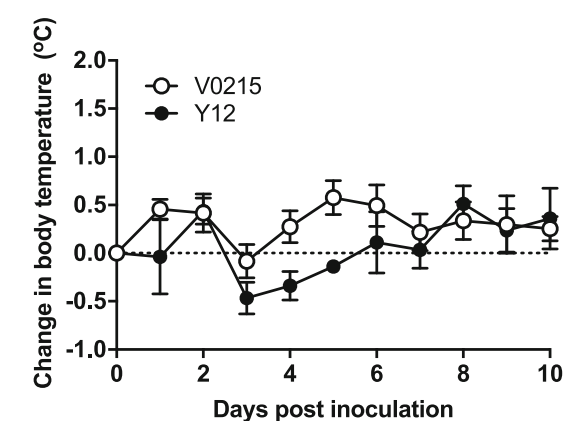

C

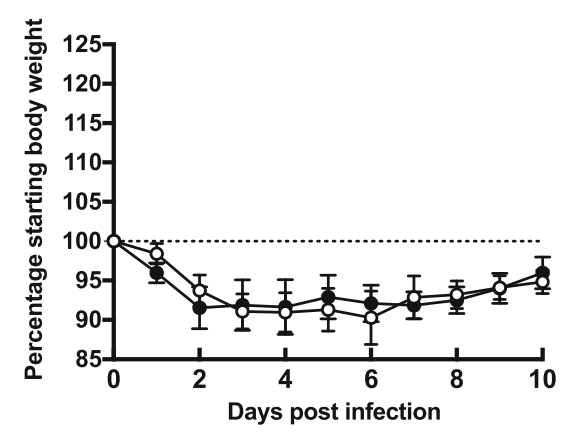

B

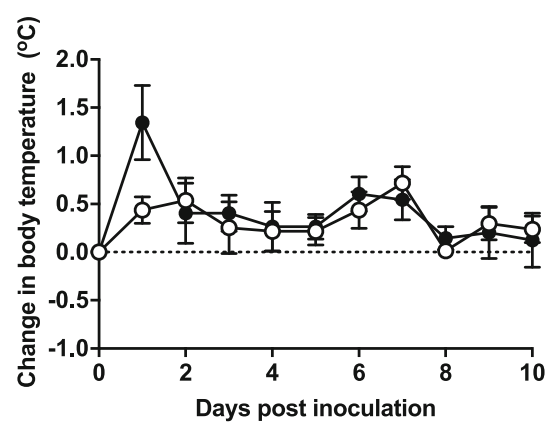

D

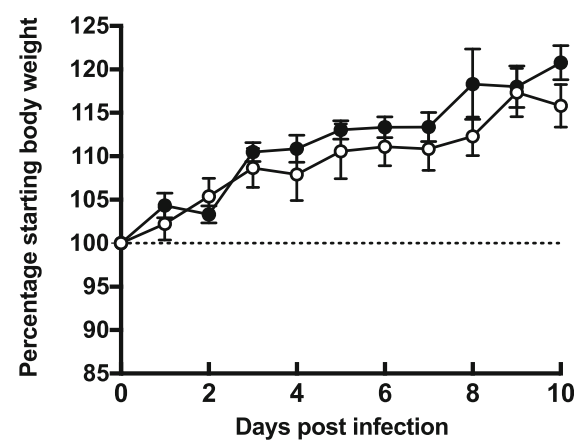

Fig. 2 Tree shrews show weight loss post inoculation with influenza B viruses but ferrets do not. Tree shrews did not show an increase in body temperature greater than $0.5^{\circ} \mathrm{C}$ above pre-inoculation (a) and up to $10 \%$ body weight loss (c) post inoculation with influenza B viruses. Ferrets inoculated with B/Guangzhou/12/2016 showed a mean body temperature increase of over $1^{\circ} \mathrm{C}$ at one day post inoculation and at other timepoints temperatures were approximately $0.5^{\circ} \mathrm{C}$ above pre-inoculation (b). Ferrets did not lose weight post inoculation with either virus (d). Body temperature and weight were measured daily after inoculation with $1.0 \times 10^{6} \mathrm{TCID}_{50}$ of virus intranasally. The body temperature at 0 day was the average of five days before infection. • Yamagata strain B/Guangzhou/12/2016 (Y12), oVictoria strain B/Guangzhou/0215/2012 (V0215)

only one ferret per timepoint was used for cytokine analysis, meaning interpretation of this dataset is limited. In mouse lungs, increased amounts of IL-6, IL-10, IP-10 and TNF- $\alpha$ mRNA were detected at 2, 4 and 6DPI (Fig. 6). Y12 elicited a significantly greater expression of IL-6 at 2 and 4DPI, IL-10 at 2 and 6DPI, IP-10 at 2, 4 and 6 DPI and TNF- $\alpha$ at 6DPI compared to V0215 (Fig. 6).
Pathological changes were observed in the respiratory tracts of tree shrews, ferrets and mice inoculated with V0215 of Y12

Histopathology of respiratory tissues obtained from tree shrews inoculated by IBVs revealed rhinitis and pneumonia in all animals, evident as submucosal hyperemia and focal edema and the presence of inflammatory cells (Figs. 7, 8 and

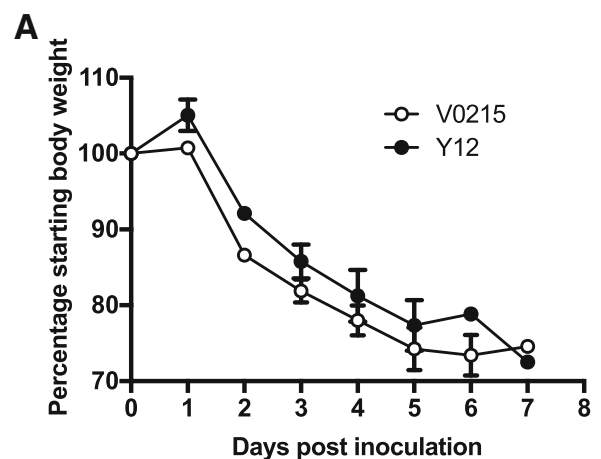

B

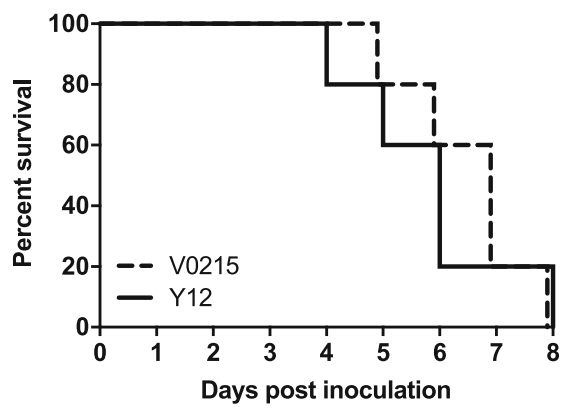

Fig. 3 Both Yamagata and Victoria lineage influenza B viruses caused 100\% mortality in BALB/C mice. Mice inoculated with either Yamagata strain B/Guangzhou/12/2016 (Y12) or Victoria strain B/Guangzhou/0215/2012 (V0215) began losing weight at two days post inoculation (a). All mice were killed by eight days post inoculation (b). • Yamagata strain B/Guangzhou/12/2016 (Y12), oVictoria strain B/Guangzhou/0215/2012 (V0215) 
Table 1 Seroconversion and $\mathrm{HI}$ titers measured in tree shrews and ferrets 21 days post inoculation with influenza B viruses

\begin{tabular}{lll}
\hline Virus & \multicolumn{2}{l}{ Number of animals seroconverted (mean and range of HI titers) } \\
\cline { 2 - 3 } & Tree shrew & Ferret \\
\hline V0215 & $5 / 5(408,40-640)$ & $5 / 5(1152,640-2560)$ \\
Y12 & $4 / 4(280,160-320)$ & $4 / 4(560,320-640)$ \\
\hline
\end{tabular}

9). Evidence of mild rhinitis in six of nine tree shrews inoculated with V0215 was also evident (Fig. 7). Two tree shrews showed evidence of moderate rhinitis and one showed severe rhinitis. Tracheitis, characterized desquamated cilia and incrassate epithelium, was evident in one mild case and one moderate case in nine tree shrews inoculated with V0215 and three mild cases in nine tree shrews inoculated with Y12. Pneumonia was also evident as pulmonary interstitial thickening filled with inflammatory cells (Fig. 7). Five mild cases, one moderate case and one severe case of pneumonia

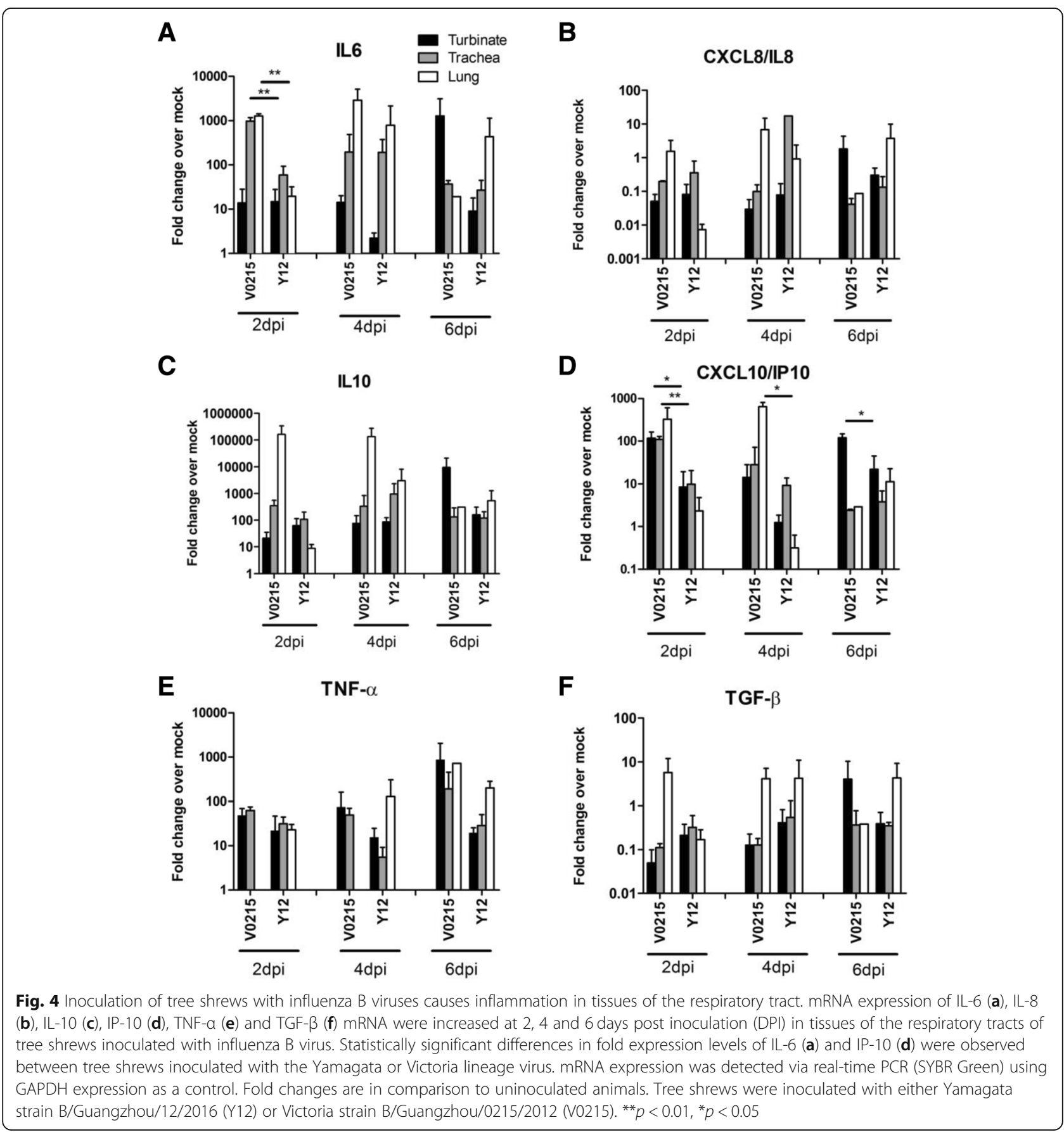




\section{A}

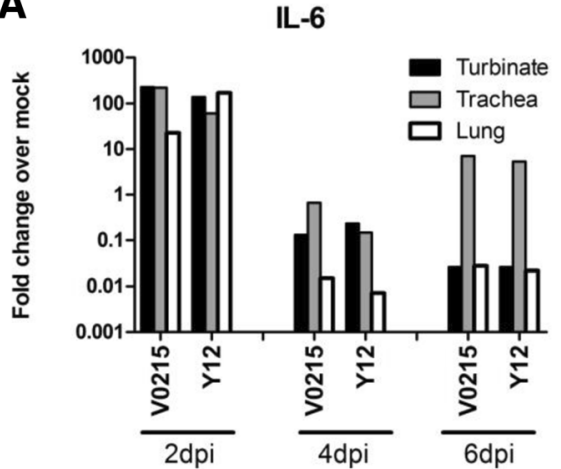

C

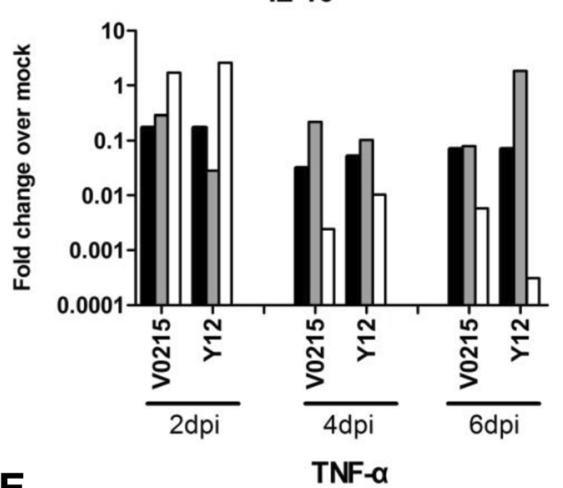

E

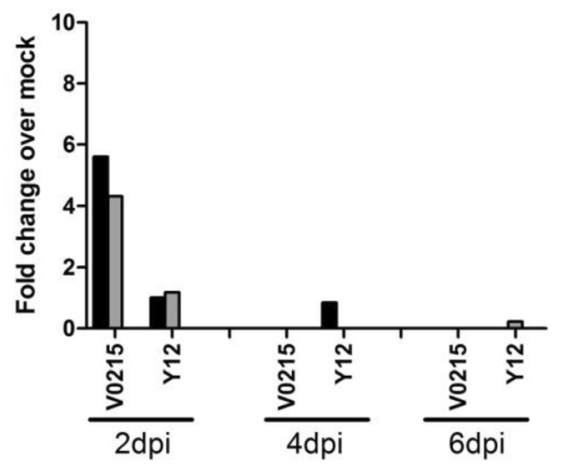

B

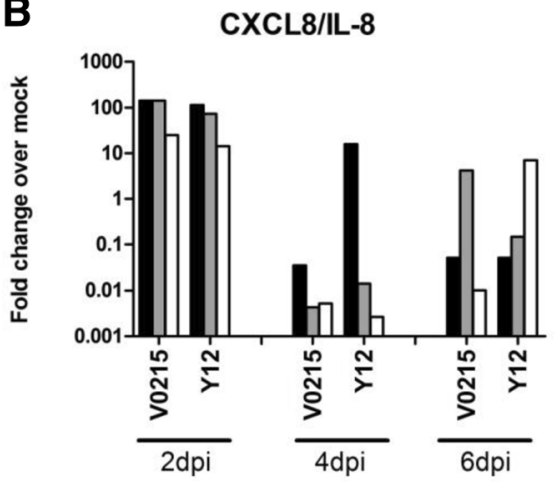

D

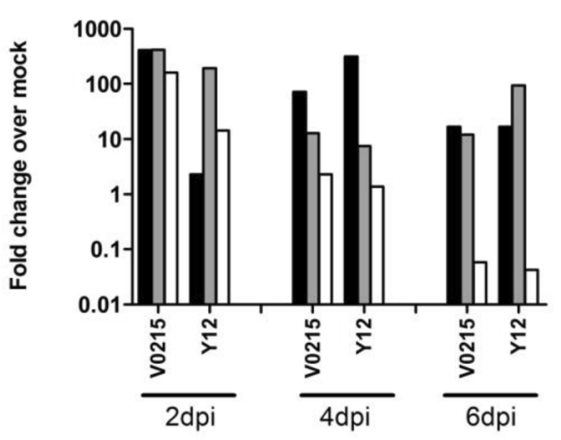

$\mathbf{F}$

TGF- $\beta$

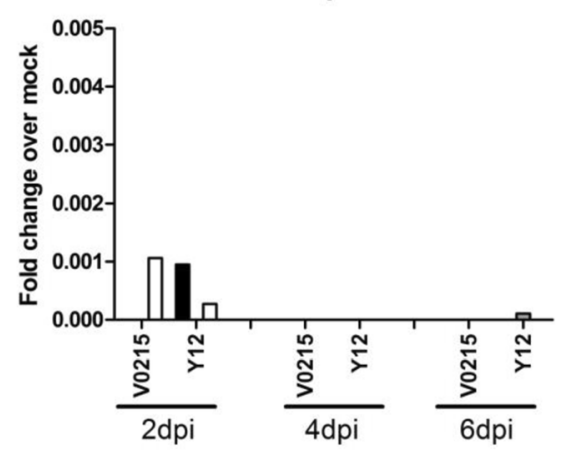

Fig. 5 Inoculation of ferrets with influenza B viruses causes inflammation in tissues of the respiratory tract. mRNA expression of IL-6 (a), IL-8 (b), IL-10 (c), IP-10 (d), TNF-a (e) and TGF- $\beta$ (f) mRNA were increased at 2, 4 and 6 days post inoculation (DPI) in tissues of the respiratory tracts of ferrets inoculated with influenza B virus. mRNA expression was detected via real-time PCR (SYBR Green) using GAPDH expression as a control. Fold changes are in comparison to uninoculated animals. Ferrets were inoculated with either Yamagata strain B/Guangzhou/12/2016 (Y12) or Victoria strain B/Guangzhou/0215/2012 (V0215)

were observed in seven tree shrews inoculated with V0215. Two mild, three moderate and two severe cases of pneumonia were observed in nine tree shrews inoculated with Y12. Overall, it appeared that Y12 caused more severe disease in tree shrews compared to V0215.

Histopathology of respiratory tissues obtained from ferrets revealed rhinitis and pneumonia in both V0215 and Y12 inoculated ferrets at 2, 4 and 6DPI. A mild tracheitis characterized by few desquamated cilia was only evident in one Y12 inoculated ferret at 2DPI (Fig. 8). A moderate rhinitis characterized by hyperemia and inflammatory cells in the submucosal layer of the nasal turbinate was observed in Y12 inoculated ferrets at 2 and 4DPI (Fig. 8d and j, respectively) and in a V0215 inoculated ferret at 2DPI (Fig. 8a). Severe rhinitis was also observed in a Y12 inoculated ferret at 6DPI (Fig. 8p) and V0215 inoculated ferrets at 4 and 6DPI (Fig. 8g and $\mathrm{m}$, respectively). Interstitial pneumonia characterized by pulmonary interstitial thickening filled with inflammatory cells was observed in both V0215 and Y12 inoculated ferrets. Severe pneumonia was also observed in a V0215 inoculated 
A

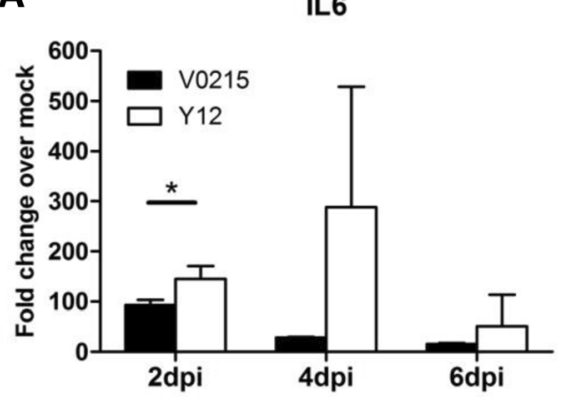

C

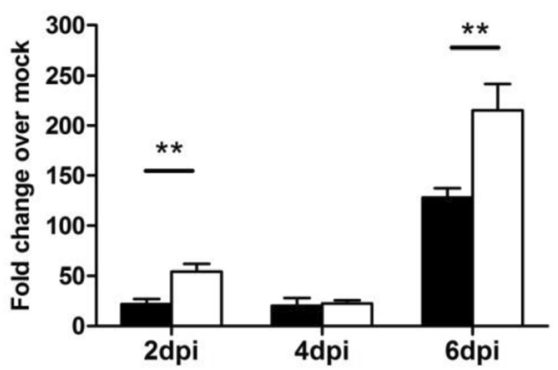

E

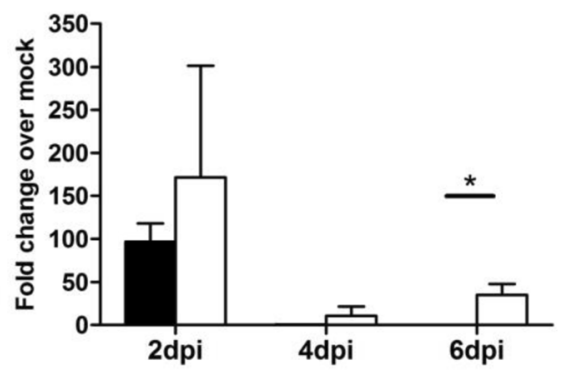

B

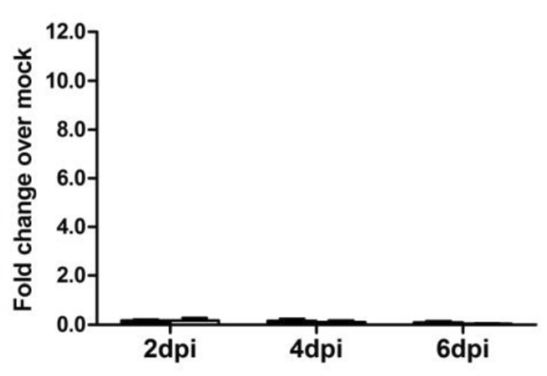

D
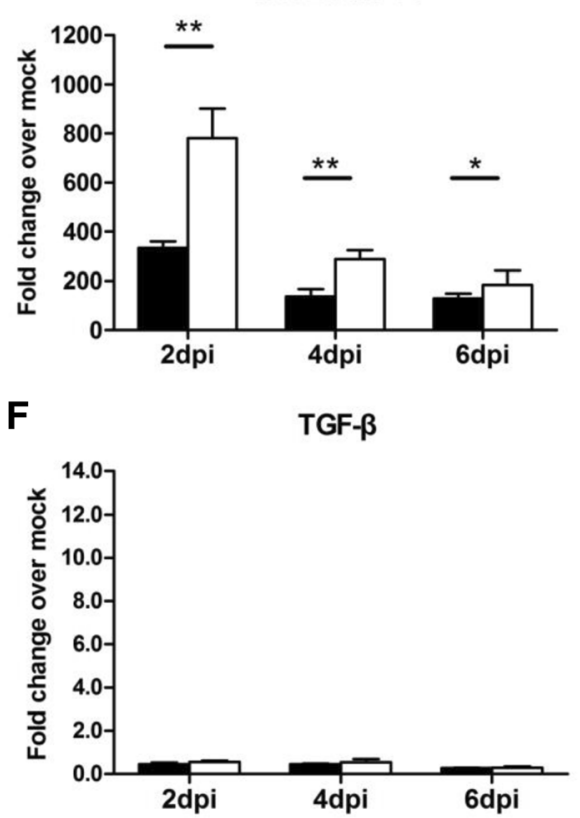

Fig. $\mathbf{6}$ Inoculation of BALB/C mice with influenza B viruses causes inflammation in tissues of the respiratory tract. mRNA expression of IL-6 (a), IL-8 (b), IL-10 (c), IP-10 (d), TNF-a (e) and TGF- $\beta$ (f) mRNA were increased at 2, 4 and 6 days post inoculation (DPI) in tissues of the respiratory tracts of mice inoculated with influenza B virus. The Yamagata strain B/Guangzhou/12/2016 elicited greater increases in the expression of IL-6 at two days post inoculation (DPI) (a), IL-10 at two and six DPI (c), IP-10 at all time points (d) and TNF-a at 6DPI (e). mRNA expression was detected via realtime PCR (SYBR Green) using GAPDH expression as a control. Fold changes are in comparison to uninoculated animals. Mice were inoculated with either Yamagata strain B/Guangzhou/12/2016 (Y12) or Victoria strain B/Guangzhou/0215/2012 (V0215)

ferret at 2DPI (Fig. 8c), although in inoculated ferrets examined at 6DPI, evidence of inflammation was mild (Fig. $8 \mathrm{i}$ and o). Moderate pneumonia was observed in Y12 inoculated ferrets at 2, 4 and 6DPI (Fig. 8f, 1 and r, respectively). Overall, there were no obvious differences between ferrets inoculated with V0215 or Y12.

After inoculation with Y12, interstitial pneumonia was observed in all but two mice (Fig. 9). Of those 15 mice inoculated with V0215, 9 mice showed severe pneumonia; five at 4DPI and four at 6DPI. Four mice showed pneumonia of moderate severity; three at 2DPI and one at 6DPI. Two mice showed pneumonia of mild severity at 2DPI. Of mice inoculated with Y12, 1 of 15 was killed at 5DPI. Three mice showed severe pneumonia, two at 4DPI and one at 6DPI. Five mice showed moderate pneumonia; two at 2DPI, two at 4DPI and one at 6DPI. Overall, V0215 appeared to cause a more severe disease in mice compared to Y12.

\section{Discussion}

As one of the major causes of seasonal influenza, IBV seriously threatens human health. As vaccines and antiviral compounds are the main interventions against IBV, it is essential to have animal models that replicate human disease to use in their development. We previously inoculated mice with IBVs isolated from clinical samples and identified two IBVs capable of causing lethal infections in mice without adaptation; the Victoria-like virus B/Guangzhou/0215/2012 (V0215) and the Yamagata-like 


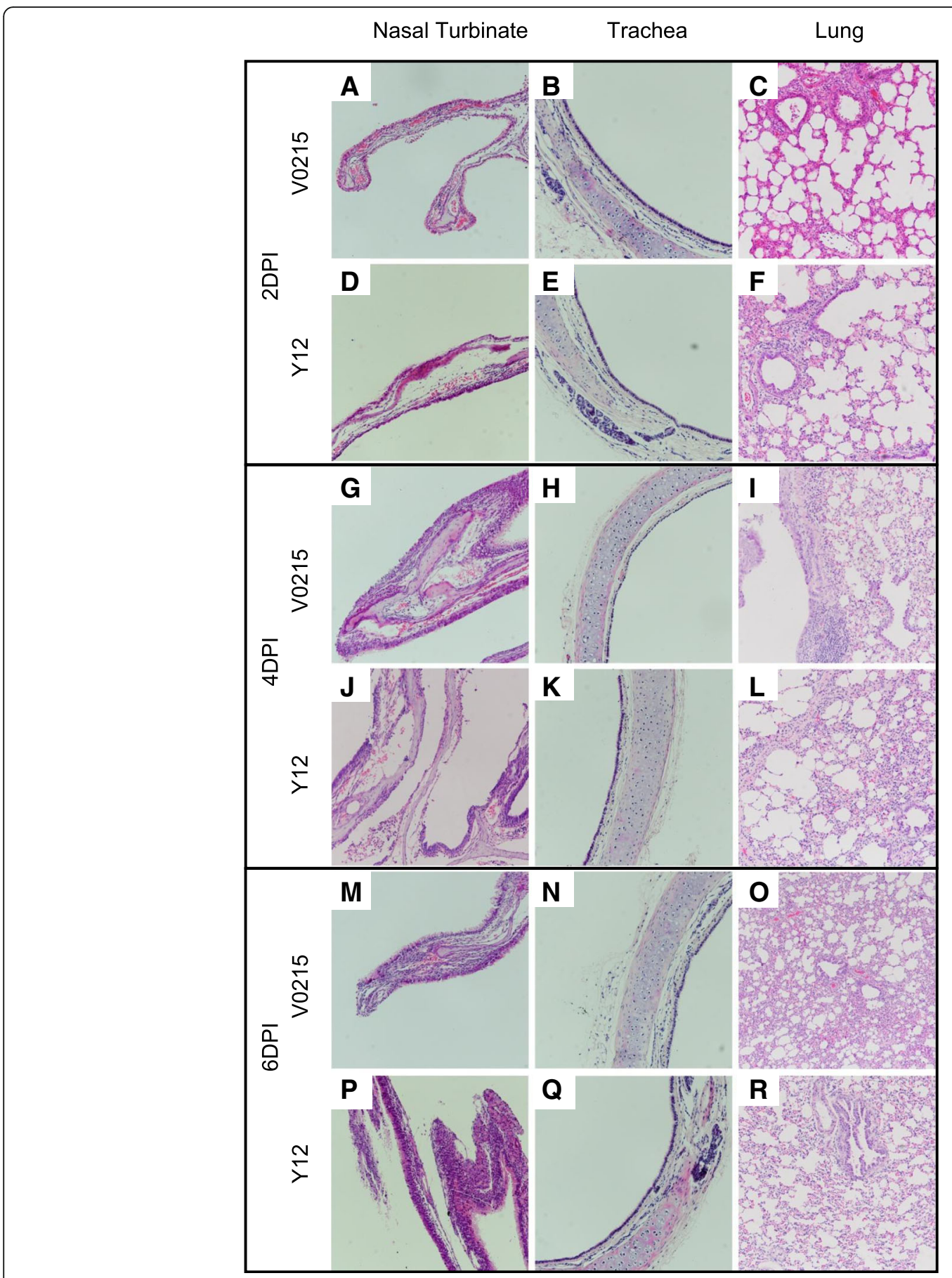

Fig. 7 Influenza B virus inoculation caused pathology in the nasal turbinates, trachea and lungs of trees shrews. Nasal turbinates $(\mathbf{a}, \mathbf{d}, \mathbf{g}, \mathbf{j}, \mathbf{m}, \mathbf{p})$, trachea $(\mathbf{b}, \mathbf{e}, \mathbf{h}, \mathbf{k}, \mathbf{n}, \mathbf{q})$, and lungs $(\mathbf{c}, \mathbf{f}, \mathbf{i}, \mathbf{l}, \mathbf{O}, \mathbf{r})$ were collected at 2, 4 and 6 days post inoculation (DPI) from animals inoculated with either Yamagata strain B/ Guangzhou/12/2016 or Victoria strain B/Guangzhou/0215/2012 and stained with hematoxylin and eosin. Images were examined at magnification of $\times 200$

virus B/Guangzhou/12/2016 (Y12). In this current study, we used these viruses to compare IBV infection in tree shrews to ferrets and mice, to determine if tree shrews could be a model for future studies of IBVs.

Previously, we demonstrated that tree shrews present a useful alternative model for the study of subtype H1N1
IAV infection. Evidence of viral replication in the upper respiratory tract was observed, as were fever, nasopharyngeal secretions and inflammation. Additionally, the distribution of $\alpha 2,3$-linked and $\alpha 2,6$-linked sialic acids were similar to that found in the human respiratory tract. However, the effects of IBV in tree shrews were 


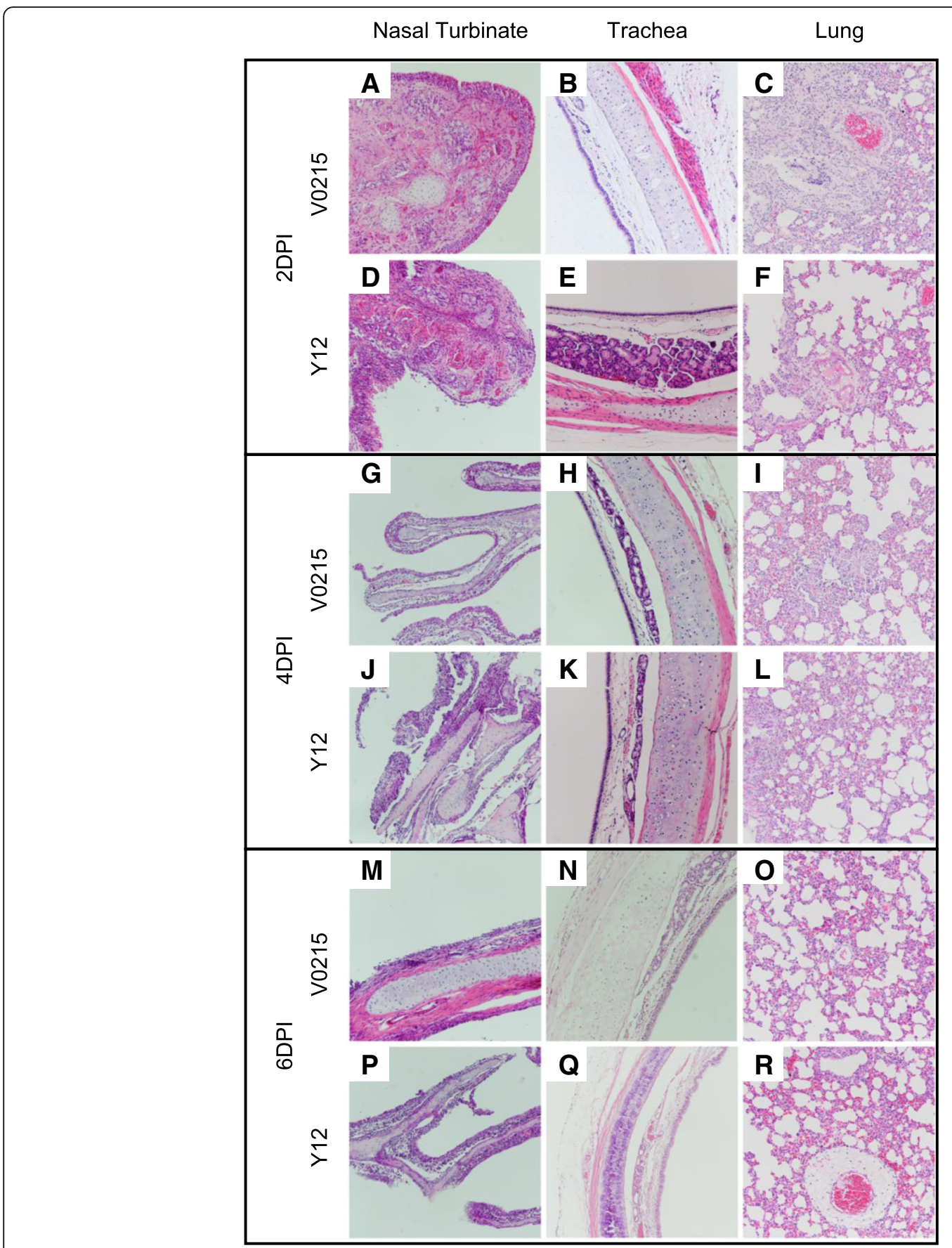

Fig. 8 Influenza B virus inoculation caused pathology in the nasal turbinates, trachea and lungs of ferrets. Nasal turbinates $(\mathbf{a}, \mathbf{d}, \mathbf{g}, \mathbf{j}, \mathbf{m}, \mathbf{p})$, trachea $(\mathbf{b}, \mathbf{e}, \mathbf{h}, \mathbf{k}$, $\mathbf{n}, \mathbf{q})$, and lungs $(\mathbf{c}, \mathbf{f}, \mathbf{i}, \mathbf{l}, \mathbf{o}, \mathbf{r})$ were collected at 2, 4 and 6 days post inoculation (DPI) from animals inoculated with either Yamagata strain B/Guangzhou/12/ 2016 or Victoria strain B/Guangzhou/0215/2012 and stained with hematoxylin and eosin. Images were examined at magnification of $\times 200$

unknown. Our results showed that Yamagata and Victoria-lineage IBVs were capable of replication in the respiratory tract of tree shrews, ferrets and mice. Clinical signs and pathological changes were also evident in the respiratory tracts of these animals. Ferrets infected with influenza B virus may have mild respiratory and systemic symptoms, including fever, runny nose, sneezing, however, these nasal and systemic symptoms of tree shrews are not as obvious as ferrets, But in other aspects, tree shrews appeared to be more sensitive to IBV inoculation compared to ferrets, as weight loss was observed in tree shrews but not in ferrets. However, the overall severity of infection in these two animal models remained mild, which is comparable to what is observed 


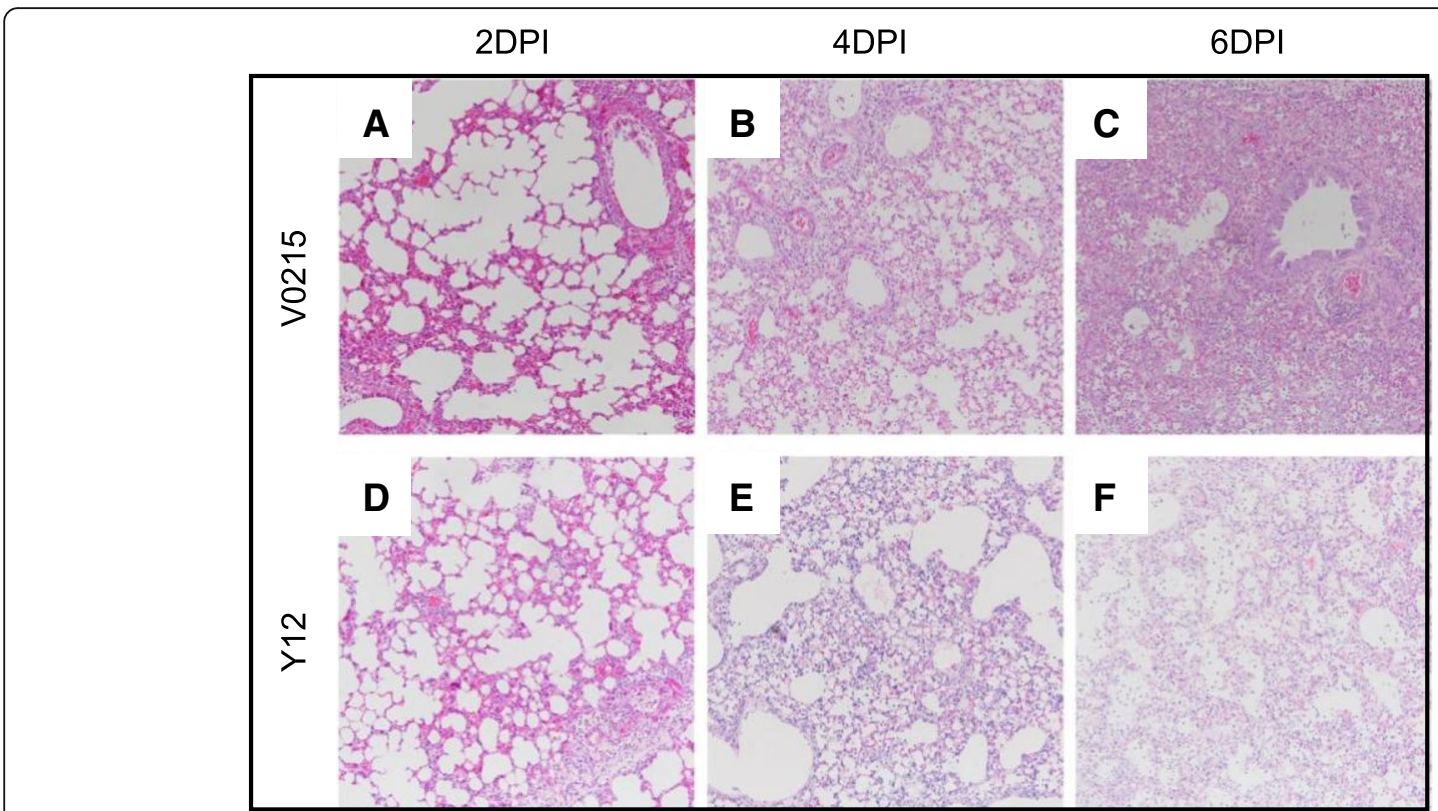

Fig. 9 Influenza B virus inoculation caused pathology in the lungs of mice. Lungs were collected at $2(\mathbf{a}, \mathbf{d}), 4(\mathbf{b}, \mathbf{e})$ and 6 (c, $\mathbf{f})$ days post inoculation (DPI) from mice inoculated with either Yamagata strain B/Guangzhou/12/2016 or Victoria strain B/Guangzhou/0215/2012 and stained with hematoxylin and eosin. Images were examined at magnification of $\times 200$

in humans. In the course of this study, we observed that the main symptoms in mice infected with influenza virus include ruffled fur, arched back, shortness of breath and malaise. From the analogue comparison of symptoms after virus infection, tree shrews and ferrets were superior to mice as a model for human IBV infection. Effective replication of viruses in host is the main pathogenic mechanism. Previously, it was considered that unadapted influenza B virus could not be infected or replicated effectively in laboratory animals such as mice $[16,17]$, because influenza $B$ virus exhibit a limited host range and lacked natural animal hosts $[18,19]$. In this study, however, tree shrews, ferrets and mice were susceptible to two lineages of unadapted human influenza B (V0215 and Y12). The results showed that the respiratory tract of these animals infected with influenza B virus could replicate the virus effectively, which the peak of virus replication was 2 days after infection. The peak and period of respiratory viral shedding of tree shrews infected with influenza B virus were similar to those of ferrets, and similar to the respiratory viral shedding symptoms of human influenza diseases,such as viral shedding time of duration and peak time of viral shedding. $[20,21]$, whereas the viral shedding cycle of mice increased significantly, and there were still high viral titers in the lung tissues until 6DPI. We also found differences in susceptibility of three animals to above two influenza B viruses. There were no significant differences in titers between V0215 and Y12 inoculated ferrets, however, viral titers were significantly higher in tree shrews and mice inoculated with Y12 compared to V0215 at both 2 and 4DPI. This difference may be related to the different virulence and tissue tropism of V0215 and Y12, as well as host differences [22, 23]. Although there was no difference in viral titers between tree shrews and ferrets infected with Y12, viral titers were detected at 6DPI in tree shrews but not in ferrets. Histopathology of respiratory tissues obtained from tree shrews inoculated by IBVs revealed rhinitis and pneumonia in all animals. Because Y12 has longer viral shedding time and higher viral titer in tree shrews, it appeared that Y12 caused more severe disease in tree shrews compared to V0215 by pathological examination.

The antibody response of hosts infected with influenza virus is an important immune and epidemic prevention mechanism. Highly virulent influenza virus strains produce higher antibody titers, which are related to the severity of influenza infection [24, 25]. A survey of serum antibodies against influenza viruses in Pennsylvania showed that the titers of antibodies in most people infected with A/California/7/2009 (H1N1) and B/Brisbane/60/2008 (B/Victoria) ranged from 0 to $1: 640$ and $0-1: 640$, respectively. A few patients infected with H1N1 virus can reach 1:1280 in some months, some even greater than 1:2560 [26], Tree shrews and ferrets infected with IBV 0215 (B/Victoria) and 12 (B/Yamagata) can seroconvert, indicating that tree shrews have certain similarities in adaptive immunity with humans and ferrets, and could therefore be used in the development and research of new vaccines. 
The immune response formed by cytokines and chemokines is the first line of defense against influenza virus infection and the key factor of the immune pathogenesis of influenza virus infection in human and animal models [27]. Moderate inflammation can inhibit the replication and transmission of influenza virus and prevent the development of disease, while excessive inflammation is closely related to clinical severe pathological injury [28]. Especially in some highly pathogenic avian influenza patients, excessive inflammation can promote the imbalance of immune function and the excessive release of a large number of inflammatory cytokines, termed a "cytokine storm", leading to multiple organ dysfunction or acute respiratory distress syndrome [29]. In this study, we detected six cytokines in respiratory tract tissues of tree shrews, ferrets and mice. Elevated levels of cytokines were detected in the tissues isolated from the respiratory tract of tree shrews, ferrets and mice after infection with either V0215 or Y12 compared to the levels in the uninfected control, suggesting that infection with IBV can trigger innate immune responses in experimental animals including tree shrews. The expression of these cytokines correlated well with the pathological changes of respiratory tract tissues, as observed in similar reports using the ferret model [30, 31]. Similarly, many pro-inflammatory cytokines and chemokines such as IL-6, IL-8, IP-10, MIG, TNF- $\alpha$ and so on were significantly up-regulated in Influenza virus infection patients [32, 33]. In addition, IL-10 is an anti-inflammatory cytokine, which can strongly inhibit the synthesis of TNF- $\alpha$, IL-1, IL-6, IL-8 and other inflammatory mediators. In this study, IL-10 increased significantly in tree shrews and mice tissues, suggesting that similar to human infection, tree shrews infected with influenza can also form a feedback regulation mechanism between pro-inflammatory and anti-inflammatory responses, maintaining the balance between inflammatory and anti-inflammatory mediators in the body [34].

Therefore, considering their small size, ease of handling and reduced cost of the tree shrews in comparison to ferrets, they present as a useful model for the study of IBV infection.

\section{Conclusion}

Our data shows that two human IBVs, Y12 and V0215, which cause lethal infections in mice, can replicate and cause pathological changes in the respiratory tract of ferrets and tree shrews. Further, we observed that tree shrews display clinical signs such as fever, weight loss and nasal exudates following IBV inoculation that were either absent or milder in ferrets. In conjunction with their ease of handling, small size and cost effectiveness, the tree shrew model represents a viable animal model for the study of human IBVs.

\section{Additional file}

Additional file 1: Table S1. The primers of ferret, tree shrew and mice. (DOCX $15 \mathrm{~kb})$

\section{Abbreviation}

BSL: Biosafety Level; DPI: Days post inoculation; HI: Hemagglutination inhibition; IAVs: Influenza A viruses; IBVs: Influenza B viruses; IVC: Independent ventilation cage; MDCK: Madin-Darby canine kidney cell; $\mathrm{TCID}_{50}$ : 50\% Tissue culture infective dose; TPCK: L-(tosylamido-2-phenyl) ethyl chloromethyl ketone; V0215: Influenza virus B/Guangzhou/0215/2012; Y12: Influenza virus B/ Guangzhou/12/2016

\section{Acknowledgments}

We thank Dr. Feng Ye for gift of influenza B virus B/Guangzhou/12/2016.

\section{Funding}

This work was supported by National Natural Science Foundation of China (NSFC-81471937, NSFC-81661168012), Regional Science Fund of National Natural Science Foundation of China (NSFC - 81760379), the Yangcheng Scholars Research Fund for Guangzhou Municipal Universities (1201561571), Foundation for Medical Discipline Leader in Health and Family Planning Commission of Yunnan Province (D-2017050).

Availability of data and material

Not applicable.

\section{Authors' contributions}

ZY conceived and designed the study. XX provided the biosafety level 2 laboratory. BY and CY performed all the animal experiments and main writing. ZM and JC performed the histological examination of the tissues. All authors read and approved the final manuscript. $M Z$ and SW reviewed and edited the manuscript. JZ, YZ, RL and NZ provided the comments. All authors read and approved the manuscript.

\section{Ethics approval and consent to participate}

All animal research was approved by the Guangdong Provincial Department of Science and Technology, which complied with the guidelines of Guangdong Regulation for Administration of Laboratory Animals (2010), and the guidelines on the welfare of medical experiment animal were used in research. Animals were allowed free access to food and water and kept on a 12-h light/dark cycle, received environmental enrichment and were monitored daily for evidence of disease and changes in attitude, appetite, or behavior suggestive of illness. In cases of suffering animals were treated with anesthetic or otherwise the experiment was stopped by humane killing (excessive injection of pentobarbital sodium) with subsequent post mortem analysis.

Consent for publication

Not applicable.

\section{Competing interests}

The authors declare that they have no competing interests.

\section{Publisher's Note}

Springer Nature remains neutral with regard to jurisdictional claims in published maps and institutional affiliations.

\footnotetext{
Author details

${ }^{1}$ Department of Respiration, the First People's Hospital of Yunnan Province, Kunming, Yunnan 650032, People's Republic of China. ${ }^{2}$ The Affiliated Hospital of Kunming University of Science and Technology, Kunming, Yunnan 650032 People's Republic of China. ${ }^{3}$ State Key Laboratory of Respiratory Disease, National Clinical Research Center for Respiratory Disease, Guangzhou Institute of Respiratory Health, the First Affiliated Hospital of Guangzhou Medical University, Guangzhou, Guangdong 510120, People's Republic of China. ${ }^{4}$ Faculty of Life Science and Technology, Kunming University of Science And Technology, Kunming, Yunnan 650500, People's Republic of China. ${ }^{5}$ Medical Faculty, Kunming University of Science And Technology,
} 
Kunming, Yunnan 650500, People's Republic of China. ${ }^{6}$ State Key Laboratory of Quality Research in Chinese Medicine, Macau University of Science and Technology, Avenida Wai Long, Taipa, Macau, People's Republic of China.

Received: 26 December 2018 Accepted: 30 April 2019

Published online: 07 June 2019

\section{References}

1. Burnham AJ, Baranovich T, Govorkova EA. Neuraminidase inhibitors for influenza B virus infection: efficacy and resistance. Antivir Res. 2013;100(2): 520-34.

2. [Database] World Health Organization. Global influenza surveillance and response system (GISRS). 2018. http://www.who.int/influenza/gisrs_ laboratory/en/. 2018

3. Jackson D, Elderfield RA, Barclay WS. Molecular studies of influenza B virus in the reverse genetics era. J Gen Virol. 2011;92(Pt 1:1-17.

4. Moon JH, Na JY, Kim JH, Yum MK, Oh JW, Kim CR, Seol IJ. Neurological and muscular manifestations associated with influenza B infection in children. Pediatr Neurol. 2013:49(2):97-101.

5. Frank H, Wittekind C, Liebert UG, Siekmeyer M, Siekmeyer W, Schuster V, Kiess W. Lethal influenza B myocarditis in a child and review of the literature for pediatric age groups. Infection. 2010;38(3):231-5.

6. Hayden FG, Jennings L, Robson R, Schiff G, Jackson H, Rana B, McClelland G, Ipe D, Roberts $\mathrm{N}$, Ward P. Oral oseltamivir in human experimental influenza B infection. Antivir Ther. 2000;5(3):205-13.

7. Sato M, Saito R, Sato I, Tanabe N, Shobugawa Y, Sasaki A, Li D, Suzuki Y, Sakai T, Oguma T, Tsukada H, Gejyo F, Suzuki H. Effectiveness of oseltamivir treatment among children with influenza a or $B$ virus infections during four successive winters in Niigata City, Japan. Tohoku J Exp Med. 2008;214(2):113-20.

8. Sugaya N, Mitamura K, Yamazaki M, Tamura D, Ichikawa M, Kimura K, Kawakami C, Kiso M, Ito M, Hatakeyama S, Kawaoka Y. Lower clinical effectiveness of oseltamivir against influenza B contrasted with influenza a infection in children. Clin Infect Dis. 2007:44(2):197-202.

9. Tsukiyama-Kohara K, Kohara M. Tupaia belangeri as an experimental animal model for viral infection. Exp Anim. 2014;63(4):367-74.

10. Fan Y, Huang ZY, Cao CC, Chen CS, Chen YX, Fan DD, He J, Hou HL, Hu L, Hu XT, Jiang XT, Lai R, Lang YS, Liang B, Liao SG, Mu D, Ma YY, Niu YY, Sun $X Q$, Xia JQ, Xiao J, Xiong ZQ, Xu L, Yang L, Zhang Y, Zhao W, Zhao XD, Zheng YT, Zhou JM, Zhu YB, Zhang GJ, Wang J, Yao YG. Genome of the Chinese tree shrew. Nat Commun. 2013;4:1426.

11. Xu L, Chen SY, Nie WH, Jiang XL, Yao YG. Evaluating the phylogenetic position of Chinese tree shrew (Tupaia belangeri chinensis) based on complete mitochondrial genome: implication for using tree shrew as an alternative experimental animal to primates in biomedical research. J Genet Genomics. 2012;39(3):131-7.

12. Yang ZF, Zhao J, Zhu YT, Wang YT, Liu R, Zhao SS, Li RF, Yang CG, Li JQ, Zhong NS. The tree shrew provides a useful alternative model for the study of influenza H1N1 virus. Virol J. 2013:10:111.

13. Yang ZF, Yang CG, Wang YT, Pan SH, Jiang HM, Li RF, Guan WD. Study on the selection of mouse adaptation strain from clinical influenza B virus. Journal of the third military Medical University. 2014;36(5):446-9.

14. Reed $\sqcup$, Muench $H$. A simple method of estimating fifty per cent endpoints. Am J Epidemiol. 1938;27(3):493-7.

15. Yang Z, Wang Y, Zhong S, Zhao S, Zeng X, Mo Z, Qin S, Guan W, Li C, Zhong $\mathrm{N}$. In vitro inhibition of influenza virus infection by a crude extract from Isatis indigotica root resulting in the prevention of viral attachment. Mol Med Rep. 2012;5(3):793-9.

16. Kim E-H, Park S-J, Kwon H-I, Kim SM, Kim Y-i, Song M-S, Choi E-J, Pascua PNQ, Choi Y-K. Mouse adaptation of influenza B virus increases replication in the upper respiratory tract and results in droplet transmissibility in ferrets. Sci Rep. 2015:5:15940.

17. McCullers JA, Hoffmann E, Huber VC, Nickerson AD. A single amino acid change in the $\mathrm{C}$-terminal domain of the matrix protein $\mathrm{M} 1$ of influenza B virus confers mouse adaptation and virulence. Virology. 2005:336(2):318-26.

18. Kitano M, Itoh Y, Kodama M, Ishigaki H, Nakayama M, Nagata T, Ishida $H_{\text {, }}$ Tsuchiya H, Torii R, Baba K. Establishment of a cynomolgus macaque model of influenza B virus infection. Virology. 2010;407(2):178-84.

19. Osterhaus A, Rimmelzwaan G, Martina B, Bestebroer T. Fouchier R. Influenza B virus in seals. Science. 2000;288(5468):1051-3.
20. Carrat F, Vergu E, Ferguson NM, Lemaitre M, Cauchemez S, Leach S, Valleron A-J. Time lines of infection and disease in human influenza: a review of volunteer challenge studies. Am J Epidemiol. 2008;167(7):775-85.

21. Fritz RS, Hayden FG, Calfee DP, Cass LM, Peng AW, Alvord WG, Strober W Straus SE. Nasal cytokine and chemokine responses in experimental influenza a virus infection: results of a placebo-controlled trial of intravenous zanamivir treatment. J Infect Dis. 1999;180(3):586-93.

22. Bodewes R, Rimmelzwaan GF, Osterhaus AD. Animal models for the preclinical evaluation of candidate influenza vaccines. Expert Rev Vaccines. 2010;9(1):59-72

23. Rowe T, Banner D, Farooqui A, Ng DC, Kelvin AA, Rubino S, Huang SSH, Fang $Y$, Kelvin DJ. In vivo ribavirin activity against severe pandemic H1N1 influenza a/Mexico/4108/2009. J Gen Virol. 2010;91(12):2898-906.

24. Huang SS, Banner D, Fang Y, Ng DC, Kanagasabai T, Kelvin DJ, Kelvin AA. Comparative analyses of pandemic $\mathrm{H} 1 \mathrm{~N} 1$ and seasonal $\mathrm{H} 1 \mathrm{N1} 1, \mathrm{H} 3 \mathrm{~N} 2$, and influenza B infections depict distinct clinical pictures in ferrets. PLoS One. 2011;6(11):e27512.

25. Sym D, Patel PN, El-Chaar GM. Seasonal, avian, and novel H1N1 influenza: prevention and treatment modalities. Ann Pharmacother. 2009:43(12):2001-11.

26. Ross TM, Hairong L, Chia BS, Hill E, Weirback H, Zimmer S. Prevalence of antibodies against seasonal influenza a and B viruses during the 2009-2010 and 2010-2011 influenza seasons in residents of Pittsburgh, PA, USA. PLoS Curr. 2011;3:RRN1265.

27. Nagaoka, Y., Nosaka, N., Yamada, M., Yashiro, M., Washio, Y., Baba, K. Morishima, T., and Tsukahara, H. (2017). Local and systemic immune responses to influenza a virus infection in pneumonia and encephalitis mouse models. Dis Markers 2017, 2594231.

28. La Gruta NL, Kedzierska K, Stambas J, Doherty PC. A question of selfpreservation: immunopathology in influenza virus infection. Immunol Cell Biol. 2007:85(2):85-92.

29. de Jong MD, Simmons CP, Thanh TT, Hien VM, Smith GJ, Chau TN, Hoang DM, Chau NV, Khanh TH, Dong VC, Qui PT, Cam BV, Ha do Q, Guan Y, Peiris JS, Chinh NT, Hien TT, Farrar J. Fatal outcome of human influenza a (H5N1) is associated with high viral load and hypercytokinemia. Nat Med. 2006; 12(10):1203-7.

30. Belser JA, Maines TR, Gustin KM, Katz JM, Tumpey TM. Kinetics of viral replication and induction of host responses in ferrets differs between ocular and intranasal routes of inoculation. Virology. 2013;438(2):56-60.

31. Carolan LA, Rockman S, Borg K, Guarnaccia T, Reading P, Mosse J, Kelso A, Barr I, Laurie KL. Characterization of the localized immune response in the respiratory tract of ferrets following infection with influenza a and $B$ viruses. J Virol. 2015;90(6):2838-48.

32. Garcia-Ramirez RA, Ramirez-Venegas A, Quintana-Carrillo R, Camarena AE, Falfan-Valencia R, Mejia-Arangure JM. TNF, IL6, and IL1B polymorphisms are associated with severe influenza a (H1N1) virus infection in the Mexican population. PLoS One. 2015;10(12):e0144832.

33. Zhou J, Wang D, Gao R, Zhao B, Song J, Qi X, Zhang Y, Shi Y, Yang L, Zhu W, Bai T, Qin K, Lan Y, Zou S, Guo J, Dong J, Dong L, Wei H, Li X, Lu J, Liu L, Zhao X, Huang W, Wen L, Bo H, Xin L, Chen Y, Xu C, Pei Y, Yang Y, Zhang X, Wang S, Feng Z, Han J, Yang W, Gao GF, Wu G, Li D, Wang Y, Shu Y. Biological features of novel avian influenza a (H7N9) virus. Nature. 2013; 499(7459):500-3.

34. Blackwell TS, Christman JW. Sepsis and cytokines: current status. Br J Anaesth. 1996;77(1):110-7.

Ready to submit your research? Choose BMC and benefit from

- fast, convenient online submission

- thorough peer review by experienced researchers in your field

- rapid publication on acceptance

- support for research data, including large and complex data types

- gold Open Access which fosters wider collaboration and increased citations

- maximum visibility for your research: over $100 \mathrm{M}$ website views per year

At $\mathrm{BMC}$, research is always in progress.

Learn more biomedcentral.com/submission 